\title{
Considering the Power of the Camera in a Post-Pandemic Era of Screendance
}

Kathryn Logan, Independent artist

Keywords: camera, power, The Maya Project, Rajko, Wernimont, feminist unboxing, process, improvisation

For many years I have been practicing contemporary dance-based improvisation with cameras, theorizing-through-practice how the camera operates uniquely in relationship with dance. I created The Maya Project in 2018, a project in which my collaborators and I investigated our bodily relationships to the camera using experiments derived from our own experiences of cameras and digital media. ${ }^{1}$ Through this investigative process, we not only made some compelling visual works, but we discovered that the camera is loaded with philosophical and bodily implications.

The camera is not just another stage or the medium through which a dance is experienced. The camera is a vehicle for capturing perspective. It is a channel, a crosser of space and time, an object-participant, a powerful observer, a director of attention, and a looking practitioner. The camera is a tool of visual culture. The camera is political. The camera changes the stakes. The camera's frame is a literal tool of inclusion and exclusion.

The camera is a tool of power that can be wielded by the user, owner, and sharer. This power can be used subversively, or can participate in perpetuating dominant narratives. Former Minneapolis police officer Derek Chauvin would likely not have been convicted for murdering George Floyd in 2020 were Darnella Frazier not present with her camera, supplying visual evidence of the horrific crime. Stories get told or don't get told by way of cameras.

And this is where we dance now. Not all of us, not ubiquitously, not forever, but certainly in droves since the beginning of the Covid-19 pandemic. I wonder how the pervasiveness of the camera is changing our cultures and our bodies, and how we incorporate these shifts into the work we make. As more and more dance work is being created for and by way of the camera due to the pandemic, I wonder how artists are examining their own relationships with the camera and the stakes it carries with it. Have 
daily interactions via camera raised the stakes or lowered them? Are people more aware of the camera's power now, as they feel the energy suck of being watched on Zoom or feel a sense of connection they've been missing by speaking with someone on FaceTime? Or are they less aware of the power, as the camera becomes more quotidian?

I will point here to Jennifer Rajko and Jacqueline Wernimont's work on what they refer to as "feminist unboxing." Rajko defines this term as "a facilitatory structure for introducing a new technology into a creative process, research project, and/or collaborative working group." Rajko's work includes activities for considering the inherent risks, unknowns, and concerns around working with technologies. She suggests that this work "situates a technology within one's own lived experience."

For me, The Maya Project work unknowingly became a feminist unboxing methodology of the camera as a technology. Utilizing contemporary dance-based improvisation and cameras, my collaborators and I looked into our ambivalent feelings about and experiences with this technology and its implications in our culture and individual lives. We acted as both camera operators and movement improvisers, making constant choices about how to be in relationship to the camera: picking it up, passing it, setting it down, or doing whole sections of a dance out-of-frame, depending on how (or whether) we wanted to be seen moment-to-moment. We built scores around things that cameras do as a way of unpacking (or "unboxing") our understanding of them. ${ }^{3}$ We discovered that the way a person behaves or feels in relationship to the camera depends on who is holding the camera. We considered the stakes of recording visual information as part of the content of this work.

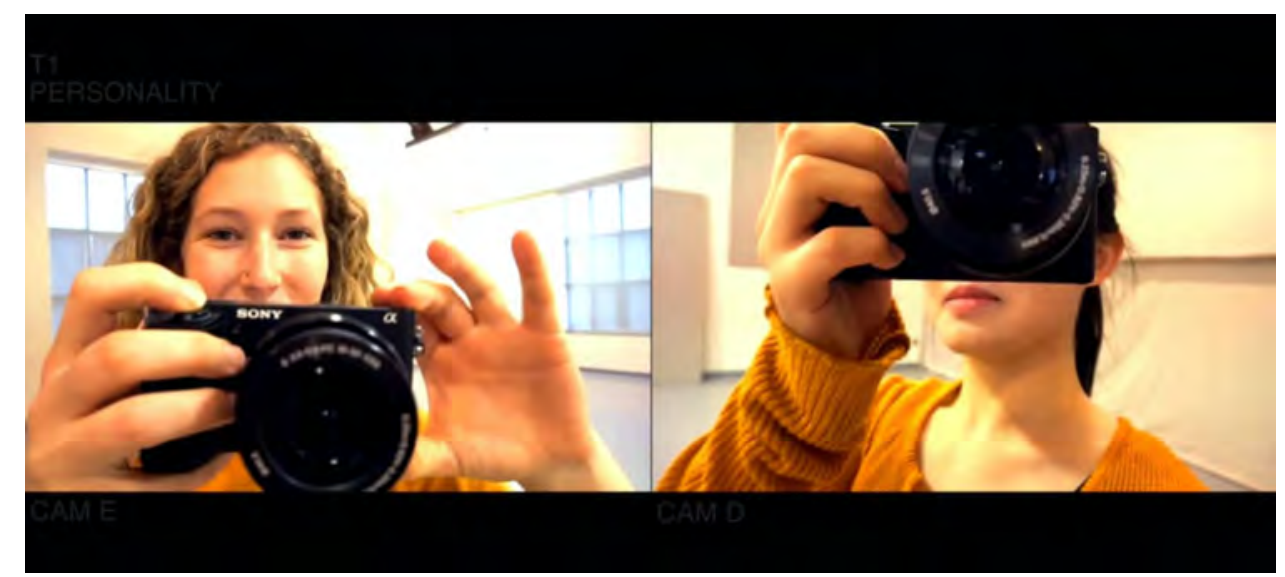

Image from The Maya Project_4_T_Personality_1, 2020. Two cameras capturing different points-of-view simultaneously in the same space. Laura Patterson by Dian Jing (left); Dian Jing by Laura Patterson (right) 


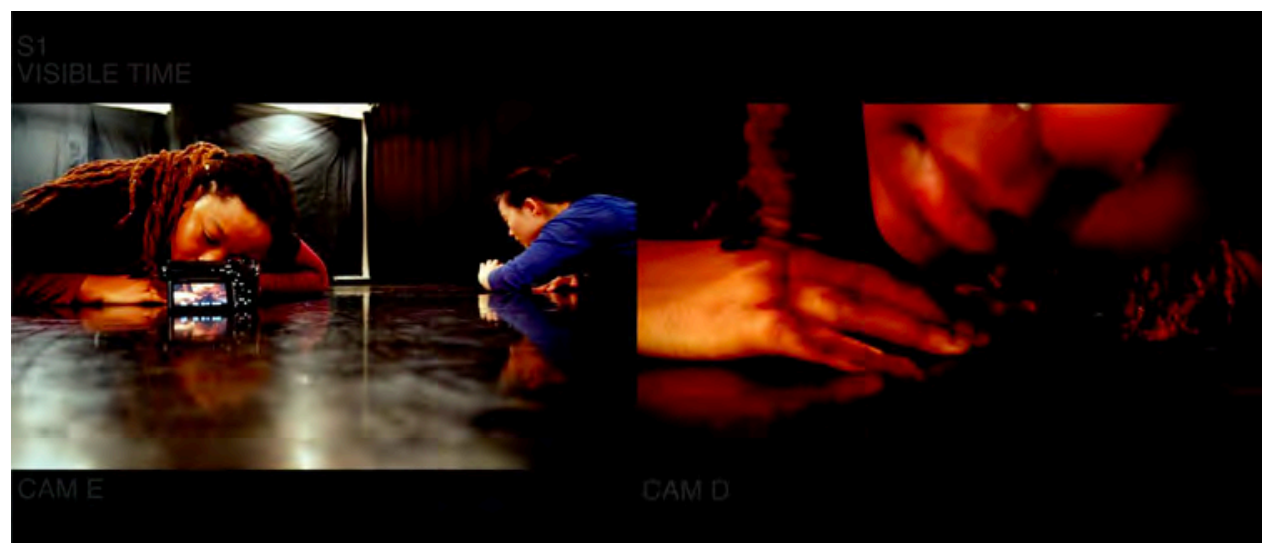

Image from The Maya Project_4_S_Visible Time_1, 2020. Davianna Green and Dian Jing by Yildiz Güventürk (left); Davianna Green by Davianna Green (right)

In this post-pandemic era of vast camera usage, I call for artists to engage in a camera "unboxing" that considers the way cameras participate in the meaning-making and context of a dance work, and considers the real life stakes of capturing and sharing visual imagery. ${ }^{4}$ Following are some questions to begin this process that have arisen from my work with The Maya Project.

How do cameras make you feel and what experiences have led you to these feelings? Hold a camera in your hand, then pass it to someone else. Take a video of them and have them take a video of you. How does it feel when the person points the camera at you? How does it feel different when they press record? How does it feel when the person holding the camera is, or is not, someone you know?

In your screendance, who wields the camera and what is their relationship to the work? If it is you, what kind of limitations does that put on your camera movement, body movement, and framing? Where does this camera come from and whose is it?

What happens to this visual / digital data and who has access to it? Was this image taken on your phone, and if so, who has access to your phone? Is this your private information? Are you sure? What is your archival process? Where do these images live?

Where is your physical attention when you are making a work with the camera? Do performers feel the same way in their bodes when the camera is pointed at them as they do when they are rehearsing or performing for a live viewership? Is this recorded or a live feed? Rehearsed or impromptu? How do each of those feel differently in the bodies of the performers?

Rajko's work with feminist unboxing is a practice through which artists can know the technologies we are using so that we might have more agency and better understanding of the effects of the contexts of those technologies on ourselves and our 
creations. For those of us who are making dance for screen, which in the post-pandemic era is only growing, the camera is an unavoidable technology. The use of the camera has and will continue to carry stakes, whether or not we consider those stakes in our creative processes. What are the various power dynamics playing out "in front of" and "behind" the camera in screendance works and what are its effects on the work we make?

\section{Biography}

Kathryn Nusa Logan is a collaborative interdisciplinary artist who utilizes experimental art practices to explore perspective, lineages, and imagined futures. Her research is based in integrated dance-with-camera works that holistically consider the camera in the making process. Through this work she is interrogating the dominant gaze by engaging in new, somatic-based practices of looking and interacting with cameras. As a video dramaturg, she works closely with directors and choreographers to utilize the camera in a way that best fits their work, interested in the camera as an innovative tool in art works beyond traditional film or dance documentation. In collaboration with the DiGS Anti-Racist Working Group at The Ohio State University (u.osu.edu/arwg), she is investing in dismantling systems of oppression in the arts and investigating how practices in somatics, dance therapy, and trauma can be activated in dance education to address systemic oppression in the body. She holds a BFA from the University of North Carolina School of the Arts and an MFA from The Ohio State University.

Email: kathrynnusalogan@gmail.com

Website: kathrynnusalogan.com

\section{Notes}

${ }^{1}$ The Maya Project is an iterative contemporary dance-based investigation of dance with camera. This work has been presented as video art installation and live performance with wireless live-feed projection, and is continually used in a workshop setting as a lens through-which to consider the camera's power and unique qualities as a technology. The Maya Project was created in collaboration with many people, most notably Davianna Green, Yildiz Güventürk, Dian Jing, Brianna Johnson, Claire Melbourne, 
Katherine Moore, and Laura Patterson. The full list of collaborators to date is available at kathrynnusalogan.com

${ }^{2}$ From "Feminist Unboxing" on Rajko's website http://www.jessicarajko.com/feministunboxing

${ }^{3}$ Humans use cameras to do things like preserve a moment, capture a sensation, or revisit a visual scene. Cameras are used to connect people across space and time, to communicate something, or to surveil. In a video vignette entitled "Preserving a Moment," we practiced conjuring the felt-sense of the desire to keep a visual memory. We identified what kinds of moments this might happen in: a moment in childhood, a wedding or graduation. We identified a sense of nostalgia in the desire to preserve a moment. Through dance improvisation, we practiced capturing visual moments, either through the camera or through our own visual field and memory. We found that this desire, nostalgia, and seeking of moments to preserve created a unique sensibility of calm attentiveness, which then informed the movement vocabulary for this section moving forward, for both the dance and the movement of the camera.

${ }^{4}$ Additionally, I suggest that we apply feminist unboxing methodologies to the platforms we use to share material: TikTok, Vimeo, YouTube, Zoom, Instagram... Each of these platforms brings their own cultures and practices of viewership, the same way live venues bring different practices of viewership: cinema spaces, proscenium theaters, outdoor concert stages, etc.

\section{References}

"About The Maya Project." KATHRYN NUSA LOGAN. Accessed 4 March 2021. https://lanusaspace.com/about-the-maya-project/

"Feminist Unboxing." Jessica Rajko. Accessed 4 March 2021. 